\title{
Endurance Life of Aging Aircraft Components Predicted by Conductivity Changes in Aluminium 2024
}

\author{
Jack A. Soules \\ Surface Stress Technology Inc. Cleveland, OH, USA
}

\begin{abstract}
In previous papers 1, 2 at the 6th and 7th International Conferences on Shot Peening, and in the technical literature 3 , we have described in detail our method of using eddy currents to compare precisely the electrical conductivity in a shot-peened metal surface to that of a similar, unpeened, surface. Encouraged by these results we began a research program to see if the surface conductivity of an ordinary aluminum plate was affected by the fatigue effects of repeated heavy loading. Since fatigue produces work hardening and other metallurgical effects we expected these effects to be observable in the conductivity. Further, in a bent specimen the stress is largest right at the surface where our technique is most sensitive.

We constructed a machine which cyclically deformed a plate of 2024 aluminum to a maximum surface stress of about 25KSI(170 megapascals) tension and about $15 \mathrm{KSI}(103$ megapascals) compression. The driving motor was turned off after each 30,000 cycles to permit us to make the surface conductivity measurements. The specimen was notched on two edges to insure that the peak stress and ultimate failure would occur in the area under observation. A photograph of the apparatus is shown in Figure 1.
\end{abstract}

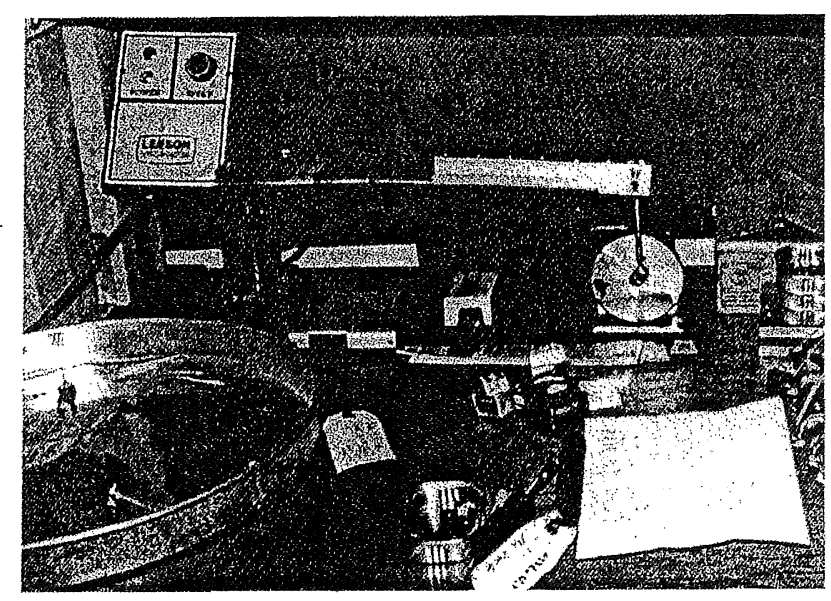

Indeed, we did find a significant variation in surface conductivity. The first twenty or thirty thousand cycles showed a progressive decrease in surface conductivity, presumably due to an increase in residual stress produced in the sample. After some thousands of cycles, however, the conductivity rather suddenly increased, tending toward the original state. The accumulated 
internal energy was evidently relieved as the material relaxed. Then the cycle repeated, building up residual stress (lower conductivity) and relaxing. Because the sawtooth conductivity cycle was unpredictable (the episodes of relaxation occurred randomly, after some tens of thousands of cycles) it could hardly be used to predict the point of failure.

An example of the surface conductivity behavior exhibited by one sample is shown in Figure 2. The data have been modified by a mathematical transformation that makes the conductivity changes more transparent. The original data, shown in Figure 3, are difficult to interpret.

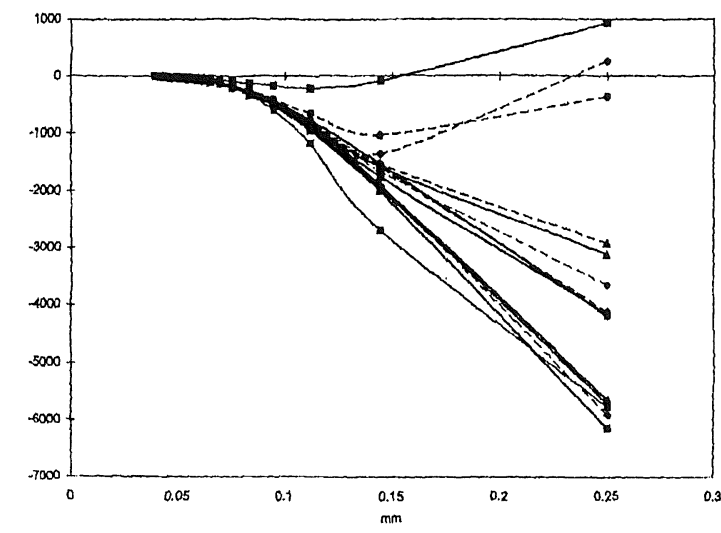

Thel 2-0 reve $\rightarrow-$ Tos: 100K $\rightarrow-$ Tes: 110KA $\because-$ Toat $130 \mathrm{~K}$ - Test 140K $\rightarrow-$ Toun 1800 - -Teat $170 \mathrm{~K}$ $\rightarrow$ Toot $180 \mathrm{~K}$ $-4-\operatorname{Tan} 190 \mathrm{~K} 2$ $-\$-$ Youl $200 k 3$ $-0-\operatorname{tent} 210 \mathrm{kz}$ $\rightarrow-$ Teal 220K

Figure 2. Changes in Conductivity due to Fatigue in 2024 Aluminum

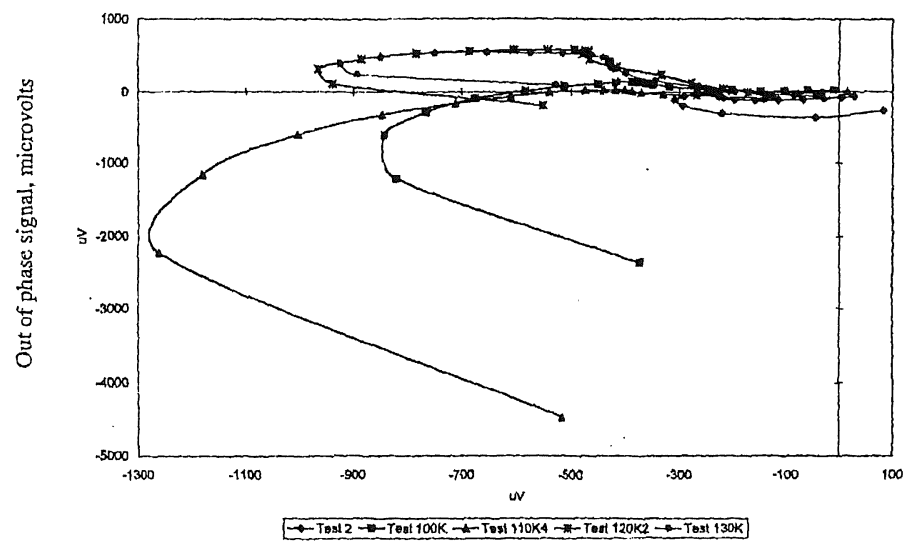

In phase signal, microvolts

Figure 3. Phasor Data, Shot-peened Aluminum Fatigue Experiment 
Educated by this experience we began a new series of experiments. This time we prepared a specimen of aluminum that had been shot-peened to approximately a 9A intensity per MIL 13165C. This results in a distribution of compressive stress to a depth of about $0.25 \mathrm{~mm}$, with the greatest stress just below the surface, diminishing to neutral at 0.30 to $0.37 \mathrm{~mm}$.

Figure 4 shows the results of this experiment. The residual stress in the surface produced a significant increase in resistivity compared to the standard, unpeened, specimen. The specific units on the ordinate of the graph are an artifact of our data algorithm. The units on the abscissa are in millimeters of depth, corresponding to the standard depth of penetration at each frequency used. The parameter identifying each curve is the number of stress cycles experienced by the sample at the time it was measured.

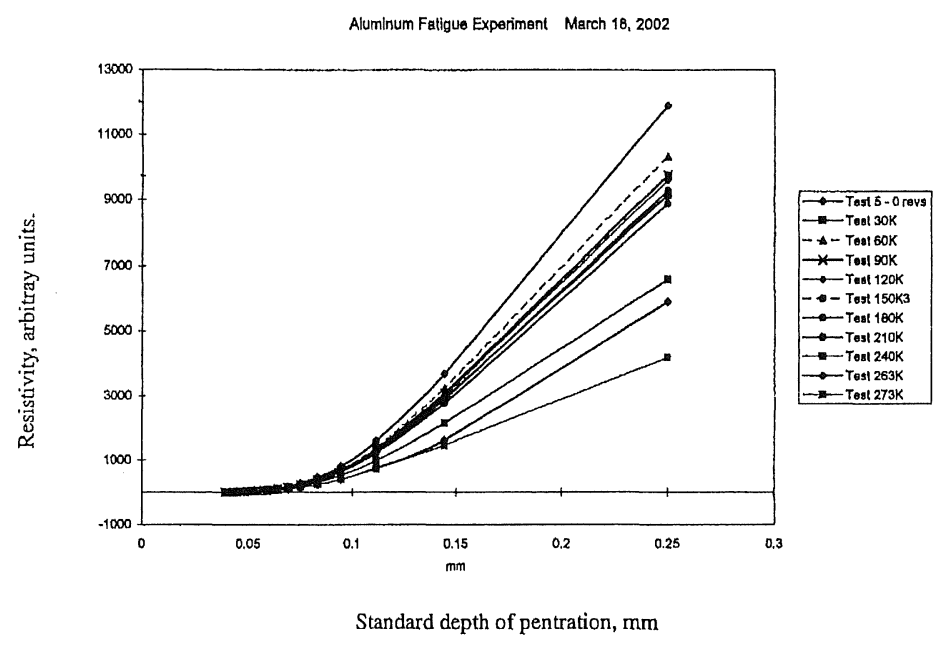

Figure 4. Shot-peened Aluminum Fatigue Experiment

Data for the uppermost curve were taken before the cyclical stress was applied and clearly shows the effect of the 9A shotpeening. The surface conductivity increased markedly at 30,000 cycles and then decreased again at 60,000 cycles. This was similar to the behavior observed in the unpeened sample. After 60,000 cycles the surface conductivity continued to increase slightly, remaining nearly constant from 90,000 to 150,000 cycles. A rapid decrease began at about 150,000 cycles and continued to 273,000 cycles, at which point the experiment was discontinued. Large cracks were visible under the microscope. The surface resistivity caused by shot-peening had declined to about $30 \%$ of its initial value at the time of failure. More importantly, the 150,000 cycle data, which represents about $55 \%$ of the ultimate fatigue life, show the resistivity down about $50 \%$ from its initial value. This would be a good point to declare the specimen "unsafe".

We intend to continue these experiments with other aluminum alloys as well as specimens of steel, titanium, and the nickelbased alloys. 


\section{REFERENCES}

1. Non-destructive Residual Stress Measurement Using Eddy Current, Hong Chang, Fred C. Schoenig, and Jack A. Soules, Sixth International Conference on Shot-Peening, San Francisco, CA, 1996.

2. Advances in Eddy Current Measurement of Residual Stress, D. Barac; W. Katcher., and J. Soules, Seventh International Conference on Shot-Peening, Warsaw, Poland, 1999.

3. Eddy Current Offers a Powerful Tool for Investigating Residual Stress and other Metallurgical Properties, Hong Chang, Frederick C. Schoenig, Jr., and Jack A. Soules, Materials Evaluation, Vol. 57, No. 12, Dec. 1999. 